\title{
Capital Humano e Crescimento Econômico: um Reexame da Abordagem Minceriana para Grupos de Países
}

Almir Bittencourt da Silva*

Emerson Marinho**

Resumo: O capital humano é reconhecidamente considerado na literatura econômica como um fator chave para o crescimento econômico. Conforme a abordagem neoclássica, o capital humano deve ser considerado como um fator de produção adicional no processo produtivo. Assim, o processo de crescimento econômico é explicado por sua acumulação. O objetivo deste artigo é estimar a contribuição do capital humano no processo de crescimento econômico utilizando uma amostra de 68 países, no período de 1960 a 1990, considerando amostras de subgrupos de países segundo seu nível de desenvolvimento. A análise empírica é implementada utilizando a abordagem da função de produção do tipo minceriana. Os resultados obtidos mostram que os dados são consistentes com a abordagem Minceriana na modelagem do capital humano.

Palavras-chave: Produtividade, Capital Humano, Função de Produção Minceriana.

Abstract: Human capital is recognised as a key factor of growth in the economic literature. According to the neoclassical approach, human capital is to be considered as an aditional input in production function. The process of growth is explained by its accumulation. Objective of this paper is to estimate the contribution of the human capital to the process of growth in a sample of 68 countries observed over the period 1960-1990, and separeted according to its development level. The empirical analysis is performed using specification through the Mincerian production function. The results obtained show that the data support the Mincerian approach to modelling of human capital.

Keywords: Productivity, Human Capital, Mincerian Production Function.

JEL Classification: $\mathrm{O} 15, \mathrm{O} 40, \mathrm{O} 47$

\section{Introdução}

O ressurgimento de pesquisas teóricas e empíricas sobre crescimento econômico foi significativamente influenciado pelo traba-

\footnotetext{
* Professor do Curso de Pós-Graduação em Economia - CAE da UFC. E-mail: almir_eco@ufc.br. Recebido em 22 de junho de 2007. Aceito em 18 de setembro de 2007.

** Professor-Doutor do Curso de Pós-Graduação em Economia - CAEN
}

Silva, A.B. Capital Humano e Crescimento Econômico... 
lho de Romer (1986), que introduziu uma nova concepção acerca do processo de crescimento das economias no longo prazo. Lucas (1988), por sua vez, inspirado na teoria do capital humano de Gary Becker (1964), concebeu importantes modificações nas hipóteses básicas do modelo de Solow (1956), impulsionando definitivamente na direção de um novo paradigma em modelos de crescimento. A idéia central incorporada nos modelos de crescimento endógenos, iniciados com Romer e Lucas, decorre da eliminação dos rendimentos decrescentes no processo de produção, em nível da economia agregada, em face das externalidades geradas pela atuação das firmas individualmente, sendo o capital humano uma de suas principais fontes impulsionadoras.

Fundamentadas em grande parte nos trabalhos teóricos iniciais da nova teoria do crescimento, várias pesquisas empíricas têm-se voltado para o exame da contribuição do capital humano no processo de crescimento. Vasta pesquisa relacionada ao desenvolvimento de dados sobre o padrão educacional de um número significativo de países (BARRO E LEE, 1993, 1996, 2000) e sobre os retornos da educação (PSACHAROPOULOS, 1994, 1995) tem permitido modos alternativos de modelagem do capital humano no processo de crescimento.

Os resultados obtidos nesses estudos indicam que o número médio de anos de escolaridade, conforme as diferentes medidas adotadas, apresenta-se fortemente correlacionada com as taxas de crescimento da produtividade. ${ }^{1}$ Além do mais, estudos de contabilidade do crescimento apontam também para a contribuição do capital humano no crescimento da produtividade ou como fator determinante das diferenças nos níveis de produtividade dos países. ${ }^{2}$

Deve-se ressaltar, no entanto, que as investigações iniciais sobre a influência da educação sobre o crescimento econômico se deram efetivamente, de forma mais sistemática, durante os anos 60 com as publicações de Schultz (1963), sobre o valor econômico da educação, e Becker (1964) com a teoria do capital humano. As diferenças observadas no crescimento do produto e a absorção efetiva de fatores de produção têm sua explicação atribuída às melhorias observadas no fator trabalho que elevam sua capacidade produtiva

1 Vejam-se, por exemplo, Barro (1991, 1999, 2001), Barro e Sala-i-Martin (1995), Benhabib e Spiegel (1994), O`Neil (1995) e Sala-i-Martin (1997).

2 A maioria desses estudos baseia-se no modelo de crescimento de Solow (1957) em que o progresso técnico é considerado exógeno. Desse modo, a variável capital humano é considerada como um insumo adicional no processo de produção. Vejam-se, por exemplo, Bills e Klenow (2000), Hall e Jones (1999) e Wöbmann (2000). A combinação dos métodos de função de produção e índices de produtividade de Malmqüist foi adotada recentemente por Grosskopf e Self (2001). 
e geram os aumentos de produtividade, os quais se refletirão, em última instância, nos aumentos de bem-estar da população. A partir de então, o conceito de investimento em capital humano passou a ser incorporado na literatura do crescimento iniciada por Solow (1957). Uma quantidade significativa de artigos sobre a contabilidade de crescimento foi então produzida com a finalidade de quantificar a contribuição dos investimentos em capital humano para o crescimento da produtividade total dos fatores (PTF) e, assim, do crescimento econômico.

Os estudos sobre a importância do capital humano para o crescimento, em geral, procuram analisar sua contribuição por meio de estimações de funções de produção agregadas, nas quais o capital humano é incluído como um fator de produção adicional. Outra maneira é avaliar seu impacto sobre a PTF através da abordagem de números índices ou pelo resíduo de Solow (1957) com a utilização de uma função de produção Cobb-Douglas. No primeiro caso, a praxe da teoria econômica tradicional é estimar funções de produção supondo que todas as unidades produtivas são eficientes.

Este artigo reexamina empiricamente a contribuição do capital humano ao crescimento econômico para um conjunto de países com padrões de desenvolvimento bastante heterogêneos, tendo como base a especificação Minceriana da função de produção. As análises são repetidas para subgrupos de países classificados pelo Banco Mundial em países ricos, de renda média e países pobres. Na modelagem do efeito em nível do capital humano, adota-se uma função de produção do tipo minceriana por razões que serão discutidas adiante.

A contribuição do artigo reside na investigação da relevância do capital humano para o crescimento econômico considerandose a diversidade dos padrões de desenvolvimento dos países quanto às possibilidades de ganhos com a educação formal. De acordo com Psacharopoulos and Patrinos (2004) e Patrinos, Ridau-Cano, Sakellariou (2006), a evidência empírica aponta que países ricos apresentam taxa de retorno para educação menor do que a dos países em desenvolvimento.

Neste artigo utilizando-se uma metodologia diferente daqueles autores, espera-se encontrar também que a contribuição do capital humano no conjunto de países ricos, medida pela estimativa do parâmetro da referida variável, tenha magnitude inferior aos dos demais grupos de países, uma vez que aqueles já alcançaram, comparativamente, padrões de educação bem superiores, apresentando, portanto, maiores oportunidades de ganho para os países menos desenvolvidos. 
No que se segue, além desta introdução o artigo está organizado da seguinte forma: a seção dois especifica a função de produção proposta por Mincer (1974) na qual se incorpora o capital humano como mais um fator de produção; a seção três define a base de dados e as variáveis empregadas além de apresentar as estimações e análise dos resultados; a seção quatro apresenta os resultados estimados dos modelos econométricos; por fim, a última seção é dedicada às conclusões.

\section{Mecanismo de Influência do Capital Humano sobre o Crescimento Econômico}

De acordo com a abordagem de Lucas (1988), seguida pela versão neoclássica adotada por Mankiw, Romer e Weil (1992) - MRWdentre outros, o capital humano pode ser incluído na função de produção como um fator de produção adicional:

$$
Y_{i t}=f\left(A_{i t}, K_{i t}, L_{i t}, h_{i t}\right)
$$

onde, $Y_{i t}$ é o produto, $A_{i t}$ representa o estado da tecnologia, $L_{i t}$ é o trabalho, $K_{i t}$ é o capital e $h_{i t}$ é o capital humano. Os subscritos $i$ e $t$ são relativos à unidade de produção (país) e ao tempo, respectivamente.

Adota-se, neste trabalho, a especificação da função de produção expressa na forma proposta por Mincer (1974) que considera a existência de apenas um tipo de trabalho cuja habilidade varia em função do nível educacional alcançado. Essa especificação, na qual o capital humano é introduzido na função de produção na forma exponencial, foi confrontada recentemente por Ferreira, Issler e Pessoa (2004) com o modelo usado por MRW, em que o referido fator é expresso em nível. Usando Box-Cox, os mencionados autores rejeitam esta última especificação em favor da primeira em um painel de dados de 95 países em vários estágios de desenvolvimento, para o período 1960-1985. Este resultado foi determinante para a escolha da especificação da função de produção usada neste trabalho, quer para amostra ampla quer para as amostras de subgrupos de países classificados segundo os níveis de desenvolvimento, sendo que nesta última situação reside o interesse principal do trabalho.

Estima-se, então, a seguinte função de produção: 


$$
Y_{i t}=A_{i t} K_{i t}\left(\exp \left(\Phi h_{i t}\right) L_{i t} \exp (g . t)\right)^{\beta}
$$

em que, $g$ representa o progresso técnico exógeno e supõe-se que o nível de habilidade de um trabalhador com $h$ anos de escolaridade é $\exp \left(\phi h_{i t}\right)$ mais elevado do que um trabalhador sem qualquer instrução. As demais variáveis têm o mesmo significado que em (1) e os sinais esperados para as estimativas dos parâmetros $\alpha, \beta, \phi$ e $g$ devem ser todos positivos, representando respectivamente as contribuições efetiva dos fatores de produção, do capital humano e da tecnologia para a formação do produto.

Normalizando a equação (2) pelo fator trabalho, $L_{i t}$, e aplicando-se logaritmo, esta passa a ter o seguinte formato que será objeto de estimação:

$$
\ln y_{i t}=\ln A_{i}+\alpha \ln k_{i t}+\beta \phi h_{i t}+(\alpha+\beta-1) \ln L_{i t}+\beta g . t+\varepsilon_{i t}
$$

onde, agora $y_{i t}=Y_{i t} / L_{i t}$ e $k_{i t}=K_{i t} / L_{i t}$. O progresso tecnológico, $A_{i t}$, em (2) é decomposto em um componente invariante no tempo específico ao país, $A_{i}$, e no componente choque de produtividade, $\varepsilon_{i t}$ que varia conforme $i$ e $t$. Ou seja, supõe-se que a tecnologia em um dado período é acessível a todos os países, sendo que as variaçõès ocorrem devido às inovações captadas por $\varepsilon_{i t}$. Além disso, deve-se observar que, nesse formato de função de produção, o coeficiente da variável $\ln L_{i t}$ mede o seu grau de homogeneidade, ou seja, se $\alpha+\beta-1=0$, tem-se retornos constantes de escala, se $\alpha+\beta-1<0$ ou $\alpha+\beta-1>0$, tem-se retornos decrescentes e crescentes de escala, respectivamente.

Por hipótese, supõe-se ainda a seguinte especificação para o choque de produtividade $\varepsilon_{i t}$ :

$$
\varepsilon_{i t}=\rho \varepsilon_{i t-1}+\eta_{i t}
$$

Em outras palavras, o choque de produtividade, $\varepsilon_{i t}$, segue um processo autoregressivo de ordem um $(\operatorname{AR}(1))$ e os $\eta_{i t}$ são independentemente distribuídos com média zero e variância $\sigma_{\eta}^{2}$. O parâmetro $\rho$ mede a autocorrelação de primeira ordem dos resíduos $\varepsilon_{i t}$. 


\section{Dados Amostrais, Estimação e Resultados}

\subsection{Dados Amostrais}

Os dados foram extraídos das seguintes fontes: Penn World Table 6.1 (PWT 6.1),(Heston, Summers e Aten, 2002), ${ }^{3}$ World Development Indicators (WDI), fornecido pelo Banco Mundial (BIRD) e Barro e Lee (2000). Esses bancos de dados internacionais são amplamente referenciados em estudos empíricos, notadamente sobre crescimento econômico, uma vez que as informações disponíveis são sistematizadas de acordo com metodologias que possibilitam a comparabilidade entre diferentes economias. As fontes de dados e as variáveis utilizadas são as seguintes:

a) PWT6.1: as séries de produto (RGDPCH - Real GDP per capita - Constant price: chain series e RGDPWOK - Real GDP chain per worker), grau de abertura (OPENK - Openness in Constant prices), gastos do governo (Kg - Government share of RGDL - Real GDP per capita - constant price Laspeyres), população (POP), desvio dos preços em relação à PPP (P - Price level of Gross Domestic Product - US = 100), trabalho (obtido por cálculo com o uso das variáveis: RGDPCH, RGDPWOK e POP);

b) WDI: capital (KAPW - Capital per worker);

c) Barro e Lee: capital humano ( $h_{i t}$ - série relativa ao número médio de anos completados de educação formal pela população com mais de 15 anos de idade, ajustada para a freqüência anual por meio de interpolação). ${ }^{4}$

Os dados das séries acima descritas utilizadas na estimação dos modelos econométricos são em níveis. Justifica-se esse procedimento pelo fato de que o uso de preços internacionais para ajustar as diferenças do poder de compra das moedas dos vários países tende a superestimar as taxas de crescimento dos países ricos e a subestimar as dos países mais pobres. Assim, evita-se a introdução de viés nos dados e, em conseqüência, sobre as estimações (NUXOLL, 1994).

3 A Penn World Table 6.1 consiste em uma atualização, que se encontra em andamento, da versão PWT 5.6.

4 Os dados de educação de Barro e Lee são apresentados originalmente em intervalos de 5 anos. Ferreira, Issler e Pessoa (2004), que usaram a interpolação da série para transformála em freqüência anual, afirmam que, embora tal procedimento possa induzir a erros de medida no capital humano, o problema é relativamente pouco significante dada a previsibilidade do comportamento da referida variável. Ademais, realizaram estimações usando as séries originais em intervalos de 5 anos e os resultados são muito próximos aos obtidos com a variável interpolada. 
A amostra utilizada é composta de 68 países no período compreendido entre os anos de 1960 e 1990, distribuídos, conforme a classificação do Banco Mundial, da seguinte forma: 23 países ricos, 28 países de renda média e 17 países pobres, conforme a descrição na tabela 1. A amostra das variáveis é constituída de 2108 observações consideradas sob a forma de um painel de dados balanceado.

Os dados foram os mesmos usados em Bittencourt e Marinho (2004) $)^{5}$ com a adição da variável capital humano. Como alguns países da referida base de dados não dispõem de informações tanto de capital físico quanto de capital humano para o ano de 1960, notadamente os países em desenvolvimento, o tamanho de amostra ficou reduzido a 68 países. Em relação ao limite superior do período de tempo (1990) da amostra, este foi definido em função da não disponibilidade das informações sobre o estoque de capital para os países a partir deste ano.

Evitou-se, portanto, adotar um procedimento bastante comum, e de certo modo arbitrário pelas suposições feitas quanto à taxa de depreciação e informações sobre investimento, para a construção de séries de estoque de capital utilizando a técnica de inventário. Dessa forma, o cruzamento dos dados relativos às variáveis capital humano e capital físico constituiu o fator determinante na definição do tamanho da amostra de países e também do período de observação.

Tabela 1. Amostra de Países

\begin{tabular}{lll}
\hline \multicolumn{1}{c}{ Países Desenvolvidos } & \multicolumn{1}{c}{ Países de Renda Média } & \multicolumn{1}{c}{ Países Pobres } \\
\hline Autrália (AUS) & Turquia (TUR) & Indonésia (IDN) \\
Áustria (AUT) & Fiji (FJI) & Bangladesh (BGD) \\
Bélgica (BEL) & República da Coréia (KOR) & Paquistão (PAK) \\
Canadá (CAN) & Malásia (MYS) & República dos Camarões (CRM) \\
Suíça (SHE) & Papua Nova Guiné (PNG) & República Centro Africana (CAF) \\
Dinamarca (DNK) & Filipinas (PHL) & Gana (GHA) \\
Espanha (ESP) & Taiwan (TWA) & Quênia (KEN) \\
Finlândia (FIN) & Tailândia (THA) & Lesoto (LSO) \\
França (FRA) & Sri Lanka (LKA) & Malawi (MWI) \\
Reino Unido (GBR) & Ihas Maurícios (MUS) & Mali (MLI) \\
Grécia (GRC) & África do Sul (ZAF) & Togo (TGO) \\
Irlanda (IRL) & Argentina (ARG) & Uganda (UGA) \\
Islândia (ISL) & Bolívia (BOL) & Zãmbia (ZMB) \\
Itália (ITA) & Brasil (BRA) & Zimbabue (ZWE) \\
Japão (JPN) & Chile (CHL) & Honduras (HND) \\
Holanda (NLD) & Colômbia (COL) & Nicaragua (NIC) \\
Noruega (NOR) & Costa Rica (CRI) & Índia (IND) \\
Nova Zelândia (NZL) & República Domiunicana (DOM) &
\end{tabular}

5 No entanto, os objetivos desses autores são distintos do atual artigo. 
\begin{tabular}{ll} 
Portugal (PRT) & Equador (ECU) \\
Suécia (SWE) & El Salvador (SLV) \\
Estados Unidos (USA) & Guatemala (GTM) \\
Hong Kong (HKG) & Jamaica (JAM) \\
Cingapura (SGP) & México (MEX) \\
& Paraguai (PRY) \\
& Peru (PER) \\
& Trinidad and Tobago (TTO) \\
& Uruguai (URY) \\
& Venezuela (VEN) \\
\hline (23 países) & \multicolumn{1}{c}{$(28$ países) } \\
\hline
\end{tabular}

Observação: Os códigos de países são adotados pelo Banco Mundial e pela PWT 6.1

\subsection{Estimações e Resultados}

Antes da definição do método de estimação para a função de produção definida por (3), alguns testes foram realizados no painel de dados visando identificar possíveis problemas econométricos que resultassem em estimativas viesadas e inconsistentes dos parâmetros. Duas preocupações iniciais referem-se à possibilidade de ocorrência de heteroscedasticidade e correlação serial no painel. O primeiro aspecto produz resultados indesejáveis na inferência estatística e o segundo acarreta estimativas viesadas dos parâmetros. Dessa forma, procedeu-se inicialmente à verificação desses dois problemas. No exame de heteroscedasticidade testou-se a hipótese de homocedasticidade contra a hipótese alternativa de heterocedasticidade utilizando o teste da Razão de Verossimilhança ${ }^{6}$ a partir da estimação da equação (3) por Mínimos Quadrados Generalizado Factível (MQGF).

$\mathrm{Na}$ verificação da autocorrelação, adotou-se o teste proposto por Wooldridge (2002) que examina a existência de autocorrelação de primeira ordem no painel.

O passo seguinte é examinar se o modelo deveria ser estimado com efeitos fixos ou efeitos aleatórios. Utilizou-se então o teste de especificação de Hausman (1978). Os resultados de todos esses testes são apresentados na tabela 2 .

Deve-se observar que para as amostras de países nos três subgrupos foram utilizados nas estimações os mesmos testes aplicados na amostra ampla. Os resultados obtidos, para o nível de significância de $5 \%$, apontaram para a existência de heterocedasticidade e autocorrelação

6 Nesse teste, sob a hipótese nula de homocedasticidade, a variância dos resíduos da equação (3) é calculada através do estimador de máxima verossimilhança onde os resíduos são obtidos da estimação de (3) por MQGF. Idem sob a hipótese de heteroscedasticidade, mas calculando-se agora a variância dos resíduos para cada país na amostra. 
dos resíduos não só para a amostra ampla de países como também para os subgrupos de países, conforme os resultados da tabela 2. O modelo de efeitos fixos, também para o nível de significância de 5\%, foi unanimidade nos testes, determinando a adoção de variáveis dummies para capturar tais efeitos.

Tabela 2. Testes de heterocedasticidade, autocorrelação e de efeitos fixos

\begin{tabular}{c|c|c|c|c|c|c}
\hline \multirow{2}{*}{$\begin{array}{c}\text { Amostra } \\
\text { de Países }\end{array}$} & \multicolumn{2}{|c|}{ Heterocedastidade( $\left.{ }^{*}\right)$} & \multicolumn{2}{|c|}{ Autocorrelação $\left(^{* *}\right)$} & \multicolumn{2}{|c}{$\begin{array}{c}\text { Efeitos Aleatórios } \\
\text { X } \\
\text { Efeitos Fixos }\left(^{* * *}\right)\end{array}$} \\
\cline { 2 - 7 } & $\begin{array}{c}\text { Razão de } \\
\text { Verossimilhança } \\
()\end{array}$ & $\begin{array}{c}\text { Graus de } \\
\text { Liberdade } \\
(\mathrm{gl})\end{array}$ & $\begin{array}{c}\text { F- } \\
\text { Snedecor }\end{array}$ & $\begin{array}{c}\text { Graus de } \\
\text { Liberdade } \\
(\mathrm{gl})\end{array}$ & $\begin{array}{c}0^{2} \\
\text { Qui - quadrado }\end{array}$ & $\begin{array}{c}\text { Graus de } \\
\text { Liberade } \\
(\mathrm{gl})\end{array}$ \\
\hline $\begin{array}{c}\text { Amostra } \\
\text { Ampla }\end{array}$ & $2.034,06$ & 67 & 351,59 & $(1 ; 67)$ & 38,96 & 4 \\
$\begin{array}{c}\text { Países } \\
\text { Ricos }\end{array}$ & 274,43 & 22 & 21,81 & $(1 ; 22)$ & 26,33 & 4 \\
$\begin{array}{c}\text { Países de } \\
\text { Renda } \\
\text { Média }\end{array}$ & 223,98 & 27 & 9,71 & $(1 ; 27)$ & 173,77 & 4 \\
$\begin{array}{c}\text { Países } \\
\text { Pobres }\end{array}$ & 133,9 & 16 & 15,47 & $(1 ; 16)$ & 28,2 & 4 \\
\hline
\end{tabular}

(") Hipótese nula: o painel é homocedástico

${ }^{(* *)}$ Hipótese nula: inexiste autocorrelação de primeira ordem

${ }^{(* * *)}$ Hipótese nula: as diferenças nos parâmetros não são sistemáticas

Uma crítica muito comum na estimação de funções de produção refere-se à endogeneidade de alguns dos fatores de produção. Esse problema é bastante presente principalmente nas estimações no âmbito da microeconomia. $\mathrm{O}$ argumento da endogeneidade sugere que a relação entre o produto e os fatores de produção está sujeita a um processo de causalidade, pois a estimação de uma função de produção deve ser tratada como a estimação de um sistema de equações simultâneas que envolvesse também as condições de primeira ordem.

O teste de endogeneidade, em sua essência, envolve a estimação usando variáveis instrumentais para as variáveis presumivelmente endógenas, verificando se as variáveis dependentes são determinadas endogenamente no sistema ou podem ser consideradas como predeterminadas. A fim de se testar a endogeneidade, uma variável como instrumento com as características de uma variável predeterminada tem que ser estimada primeiramente. Hausman (1983) sugere o uso de variáveis endógenas defasadas do próprio modelo como 
variáveis instrumentais. ${ }^{7}$ Nesse teste as variáveis instrumentais utilizadas foram trabalho, capital e capital humano defasadas em até três períodos.

Tabela 3. Estimativas dos Parâmetros da Função de Produção

\begin{tabular}{|c|c|c|c|c|c|c|c|c|}
\hline \multirow{2}{*}{$\begin{array}{c}\text { Variáveis } \\
\text { Explicativas } \\
/\end{array}$} & \multicolumn{2}{|c|}{ Amostra Ampla } & \multicolumn{2}{|c|}{ Países Ricos } & \multicolumn{2}{|c|}{ Países de Renda Média } & \multicolumn{2}{|c|}{ Países Pobres } \\
\hline & MQO & MQGF & MQO & MQGF & MQO & MQGF & MQO & MQGF \\
\hline $\operatorname{Int}_{\mathrm{i}, \mathrm{t}}$ & $\begin{array}{c}0,4596 \\
(0,0149)\end{array}$ & $\begin{array}{c}0,4350 \\
(0,0212)\end{array}$ & $\begin{array}{c}0,6504 \\
(0,0174)\end{array}$ & $\begin{array}{c}0,5986 \\
(0,0388)\end{array}$ & $\begin{array}{c}0,6086 \\
(0,0196)\end{array}$ & $\begin{array}{c}0,5165 \\
(0,0299)\end{array}$ & $\begin{array}{c}0,2788 \\
(0,0205)\end{array}$ & $\begin{array}{c}0,2791 \\
(0,0322)\end{array}$ \\
\hline$h_{i, t}$ & $\begin{array}{c}0,0401 \\
(0,0072)\end{array}$ & $\begin{array}{c}0,0423 \\
(0,0092)\end{array}$ & $\begin{array}{c}0,0174 \\
(0,0067)\end{array}$ & $\begin{array}{c}0,0235 \\
(0,0093)\end{array}$ & $\begin{array}{l}0,0023^{*} \\
(0,0100)\end{array}$ & $\begin{array}{l}0,0072^{*} \\
(0,0140)\end{array}$ & $\begin{array}{c}0,1176 \\
(0,0212)\end{array}$ & $\begin{array}{c}0,1169 \\
(0,0286)\end{array}$ \\
\hline $\operatorname{lnLi}{ }_{, t}$ & $\begin{array}{l}-0,0812 \\
(0,0076)\end{array}$ & $\begin{array}{c}-0,1945 \\
(0,0555)\end{array}$ & $\begin{array}{c}0,2272 \\
(0,0634)\end{array}$ & $\begin{array}{c}0,2155 \\
(0,1095)\end{array}$ & $\begin{array}{c}-0,1271 \\
(0,0613)\end{array}$ & $\begin{array}{c}-0,0900^{*} \\
(0,1051)\end{array}$ & $\begin{array}{l}-0,2628 \\
(0,1285)\end{array}$ & $\begin{array}{l}-0,5002 \\
(0,1476)\end{array}$ \\
\hline \multirow[t]{2}{*}{$\mathrm{t}$} & $\begin{array}{c}0,0046 \\
(0,0009)\end{array}$ & $\begin{array}{c}0,0087 \\
(0,0014)\end{array}$ & $\begin{array}{c}-0,0017 \\
(0,0013) \\
\end{array}$ & $\begin{array}{c}0,0015 \\
(0,0023) \\
\end{array}$ & $\begin{array}{l}0,0015^{\star} \\
(0,0015)\end{array}$ & $\begin{array}{c}0,0049 \\
(0,0028)\end{array}$ & $\begin{array}{c}0,0077 \\
(0,0032)\end{array}$ & $\begin{array}{c}0,0113 \\
(0,0044)\end{array}$ \\
\hline & & 0,8567 & & 0,8259 & & 0,8721 & & 0,8131 \\
\hline
\end{tabular}

Observação:MQO e MQGF são abreviações dos métodos de estimação Mínimos Quadrados Ordinários e Mínimos Quadrados Generalizados Factível completo, respectivamente. Este último também é conhecido como estimador Prais-Wisten.

O MQO utilizado é robusto nos devios-padrão

* Não significativos nos níveis de significância usuais (10\%, 5\% e 1\%)

** Os valores entre parênteses abaixo das estimativas dos parâmetros referem-se aos desvios-padrões robustos

O teste empregado para verificar a possibilidade de endogeneidade em (3) foi o de Durbin (1954)-Wu (1973)-Hausman (1978), conhecido como teste DWH, que é definido como um teste qui-quadrado. Este procedimento apresenta uma grande vantagem em relação à versão de WH, de WU (1973) e Hausman (1978) pelo fato de apresentar performance superior quando os instrumentos usados são considerados inadequados segundo Staiger e Stock (1997). O resultado obtido foi pela rejeição do capital físico, capital humano e trabalho como variáveis endógenas (o Valor - P foi igual a 1 para esses três fatores de produção considerados).

Em face destes problemas, optou-se pela estimação da função de produção (3) na amostra ampla e nos três subgrupos de países por meio de dois métodos: o método de Mínimos Quadrados Ordinários (MQO) para estimação em painel, mas usando o estimador da variância de Eicker/Huber/White em lugar do cálculo tradicional, ${ }^{8}$

7 A versão de Durbin do teste de endogeneidade tem uma grande vantagem sobre a versão de Wu e Hausman pelo fato de apresentar performance superior quando os instrumentos usados são fracos, Staiger e Stock (1997).

8 Nessa estimação, utiliza-se o comando ivreg2, no STATA 8.1. 
e o método de Mínimos Quadrados Generalizados Factível completo (MQGF), também conhecido como estimador de Prais-Winsten, ${ }^{9}$ que leva em consideração não só a autocorrelação dos resíduos, mas também a heteroscedasticidade na estimação dos parâmetros.

Os resultados das estimações dos parâmetros da função de produção, equação (3), pelos dois métodos acima descritos e do parâmetro $\rho$ da equação (4), são apresentados na Tabela 3. Observa-se que a magnitude dos parâmetros estimados pelos dois métodos é praticamente a mesma além dos sinais esperados serem coincidentes, a menos dos sinais estimados da variável trabalho que são positivos e os da variável tempo que apresentam sinais alternados na amostra de países ricos.

$\mathrm{Na}$ amostra ampla, constata-se que o sinal positivo da estimativa da variável capital humano mostra-se de acordo com a proposição dos defensores da abordagem do capital humano influenciando o crescimento econômico por meio do processo de sua acumulação. Neste caso, o capital humano constitui um fator de produção relevante no processo produtivo.

Os resultados obtidos são também bastante próximos daqueles estimados por Ferreira, Issler e Pessoa (2004). Esses autores encontram valores para $\mathrm{a}=0,4306, \mathrm{~b}=0,4501, \mathrm{f}=0,0909 \mathrm{e} \mathrm{g}=0,0221$, e retornos de escala médio no período de - 0,1193. As magnitudes desses mesmos parâmetros, obtidas a partir da estimação dos coeficientes da equação (3), considerando como referência o método de MQGF, são de $a=0,4350, b=0,3705, f=0,1142$ e $g=0,0235$, e a medida de retornos decrescentes de escala, de acordo com o coeficiente de In $L_{i t}$, foi estimada em - 0,1945, mostrando, portanto, muitas semelhanças ${ }^{10}$ entre eles. Esses autores encontram valores para $\mathrm{a}=0.4306, \mathrm{~b}=0.4501, \mathrm{f}=0.0909 \mathrm{e} \mathrm{g}=0.0221$, e retornos de escala médio no período de -0.1193 .

Deve-se destacar, ainda, que os parâmetros das variáveis lnk e InL representam não só as elasticidades, mas medem também os retornos de escala da função (3). Assim seus valores estimados mostram que enquanto a elevação do capital aumenta o produto em todos os grupos de países, no caso do trabalho, seu aumento resulta numa queda de produto somente no grupo de países pobres e amostra

9 Todas as estimações foram realizadas com o programa STATA 8.1.

${ }^{10}$ Ferreira, Issler e Pessoa (2004) mencionam vários estudos empíricos para diferentes amostras e intervalos de tempos com variações muito pequenas em alguns desses parâmetros como, por exemplo, a elasticidade do capital a (entre 0.33 e 0.43 ) e a taxa de retorno de um ano adicional de escolaridade f (entre $6.8 \%$ na OECD e $10,1 \%$ no resto do mundo).

Silva, A.B. Capital Humano e Crescimento Econômico... 
ampla sugerindo combinações de fatores de produção com substituição de trabalho por capital. Essa relação se mantém para o grupo de países de renda média somente quando os resultados são estimados por MQO, pois o efeito do trabalho sobre o produto é não significante quando a equação (3) é estimada por MQGF. No grupo de países ricos o impacto do aumento do trabalho sobre o produto é positivo e significativo.

Em relação aos retornos de escala da função de produção, o grupo de países ricos apresentou retornos crescentes de escala enquanto que no grupo de países pobres e amostra ampla de países os rendimentos de escala estimados foram decrescentes. O mesmo se dá para o grupo de países de renda média quando o modelo (3) é estimado por MQO. No caso específico da elasticidade do capital, a estimativa aqui obtida está dentro do intervalo de valores encontrados em vários estudos internacionais. ${ }^{11}$

$\mathrm{Na}$ especificação da função de produção adotada, há um particular interesse sobre o parâmetro $\Phi$ que é interpretado como a porcentagem de crescimento na renda decorrente de um ano adicional de estudo formal, ou seja, o retorno de um ano adicional de educação formal de um indivíduo com habilidade média. Mincer (1974) estimou esse parâmetro em 10\%. A estimativa aqui obtida é de $11,42 \%$.

Nas estimações realizadas para a amostra ampla de países e para os grupos de países desenvolvidos (ricos) e pobres, todos os valores obtidos para a variável capital humano apresentam sinais esperados e significativos para o nível de significância de 5\%. Conclui-se, portanto, que não se pode rejeitar a hipótese de que o capital humano tem efeito de nível importante para o crescimento econômico destes países e, desde que os coeficientes das variáveis capital e trabalho foram significativos para esse mesmo nível, a tecnologia de produção para esses grupos de países parece assemelharse a uma Cobb-Douglas convencional. Além do mais, observa-se que os países pobres apresentam a magnitude do parâmetro estimado do capital humano maior do que dos países ricos. Por outro lado, para os países de renda média os resultados estimados se mostraram não significativos para a variável capital humano.

Conclui-se, portanto, que efetivamente o capital humano tem efeito de nível importante para o crescimento econômico dos países, sendo que o impacto nos países pobres é positivamente maior.

${ }^{11}$ Ferreira, Issler e Pessoa (2004) mencionam vários estudos empíricos para diferentes amostras e intervalos de tempos com variações muito pequenas em alguns desses parâmetros como, por exemplo, a elasticidade do capital a (entre 0.33 e 0.43 ) e a taxa de retorno de um ano adicional de escolaridade f (entre $6.8 \%$ na OECD e 10,1\% no resto do mundo). 
Esse resultado corrobora a hipótese inicial levantada na introdução e confirma as conclusões de Psacharopoulos e Patrinos (2004) e Patrinos, Ridau-Cano, Sakellariou, (2006). No entanto, no caso dos países de renda média, o parâmetro estimado do capital humano não foi estatisticamente significante, o que contraria o resultado esperado. Deve-se, contudo, observar que a amostra de países de renda média na classificação do Banco Mundial é muito heterogênea, pois incorpora países com níveis de renda que vão desde média baixa até média alta, o que pode ter contribuído para esse resultado não esperado.

Estudos recentes como os de Psacharopoulos e Patrinos (2004) e Patrinos, Ridau-Cano, Sakellariou, (2006) atribuem essas diferenças entre países à grande mobilidade do trabalho observada nos países ricos, escassez relativa de mão-de-obra qualificada para desempenhar certas atividades e fatores e características específicas do mercado de trabalho.

\section{Conclusão}

A importância do capital humano como um fator estratégico para o crescimento econômico já constitui uma tradição na literatura econômica. No entanto, ainda persistem as discussões sobre os canais pelos qual essa influência se exerce. Duas principais linhas de pesquisa, aparentemente antagônicas, predominam entre os estudos empíricos. Ambas situam-se no âmbito da teoria de crescimento endógeno, sendo que uma delas, iniciada por Lucas (1988), fortemente influenciada pela tradição do modelo neoclássico, modela o capital humano na função de produção como qualquer outro insumo do processo produtivo; a outra abordagem, devida a Nelson e Phelps (1966), associa o processo de difusão tecnológica e catch-up à disponibilidade de capital humano. Uma terceira abordagem é associada à Romer (1990), também vinculada às novas teorias de crescimento, que considera o capital humano como o elemento dinâmico e determinante do processo de inovação tecnológica.

Os resultados encontrados na estimação da função de produção mostram que o capital humano constitui um fator produtivo relevante na amostra completa de países e que os retornos da educação situam-se em torno de $11.42 \%$ ao ano. Esse valor situa-se bastante próximo ao encontrado por Mincer (1974), que o estimou em torno de $10 \%$ ao ano. Nas estimações feitas para as amostras compreendendo os países segundo seu nível de desenvolvimento, as estimativas indicam que entre os países desenvolvidos e pobres o 
capital humano apresenta sinais positivos e significativos, confirmando, assim, o resultado obtido na amostra ampla. Já no caso dos países de renda média o coeficiente estimado da referida variável não resultou significante, embora seu sinal tenha sido positivo. Em relação a este último resultado, deve-se observar, no entanto que nesta classificação o Banco Mundial inclui países com níveis de renda que vão desde média baixa até média alta. Assim sendo, esta heterogeneidade pode estar contribuindo para este resultado.

Em resumo, o resultado obtido neste artigo do efeito positivo do capital humano sobre o crescimento econômico dos países corrobora o que a literatura econômica já previra. Além do mais, esse efeito é mais intenso no grupo de países pobres quando comparado ao grupo de países ricos, resultado esse que corrobora a evidência empírica, conforme os encontros de Psacharopoulos e Patrinos (2004) e Patrinos, Ridau-Cano, Sakellariou, (2006).

\section{Referências}

AGHION, P.; HOWITT, P. Endogeneous Growth Theory. MIT Press, MA, 1999.

BARRO, J. R. Economic Growth in a Cross Section of Countries. Quarterly Journal of Economics, n. 106, p. 407-443, 1991.

. Human Capital and Growth. American Economic Review, Papers and Proceedings, n. 91, p. 12-17, 2001.

BARRO, R. J.; SALA-I-MARTIN, X. Economic Growth. New York: McGraw-Hill, 1995.

BARRO, R. J.; LEE, J. W. International Comparisons of Educational Attainment. Journal of Monetary Economics, n. 32, v. 3, p. 363-394, 1993.

. Sources of Economic Growth. Carnegie-Rochester Conference Series on

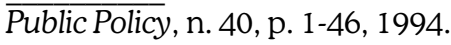

. International Measures of Schooling Years and Schooling Quality. American Economic Review, Papers and Proceedings, n. 86,p. 218-223, 1996.

. International Data on Educational Attainment: updates and implications. CID Working Paper n. 42, april, 2000. 34 p.

BECKER, G. S. Human Capital: A Theoretical and Empirical Analysis, with Special Reference to Education, third edition. Chicago and London: University of Chicago Press, 1993.

BENHABIB, J.; SPIEGEL M. The Role of Human Capital in Economic Development: Evidence Aggregate Cross-Country Data. Journal of Monetary Economics; 143-173, 1994. $34 \mathrm{p}$.

BILS, M. ; KLENOW, P. J. Does Schooling Cause Growth? American Economic Review, n. 90, p. 1160-1183, 2000.

BITTENCOURT DA SILVA, A.; MARINHO, E.; Produtividade, Crescimento Econômico e 
Dinâmica Distribucional: Um Estudo Comparado para Grupos de Países. Mimeo, CAEN, 2004

DAVIDSON, R.; MacKINNON, J. G. Econometric Theory and Methods. New York: Oxford University Press, 2004.

DUFFY, J.; PAPAGEORGIOU, C. A Cross-Country Empirical Investigation of the Aggregate Production Function Specification. Journal of Economic Growth, n. 5, p. 87120, 2000.

DURBIN, J. Errors in variables. Review of the International Statistics Institute, n. 22, p. 2332, 1954.

EICKER, F. Limit theorems for regression with unequal and dependent regressors. In: L. LeCarn; Neyman, J. Fifth Berkeley Symposium on Mathematical Statistical and Probability. Berkeley: University of California, p. 59-82, 1967.

ENGELBRECHT, H. J. International REDD Spillovers, Human Capital and Productivity. In OECD Economies: an Empirical Investigation, European Economic Review, n. 41, p. 1479-88, 1997.

FERREIRA, P. C.; ISSLER, J. V.; PESSOA, S. A. Testing Production Functions Used in Empirical Gowth Studies. Economics Letters, n. 83, p. 29-35, 2004.

HALL, R. E.; JONES, C. I. Why Do Some Countries Produce so Much More Product per worker than Others? Quarterly Journal of Economics, n. 114, v. 1, p. 83-116, 1999.

HAUSMAN, J. A . Specification Tests in Econometrics. Econometrica, n. 46, p. 12511272, 1978.

Specification and Estimation of Simultaneous Equation Models, in Zvi Griliches and M. D. Intriligator (eds), Handbook of Econometrics v. 1. Amsterdam: North Holland, 1983.

HESTON, A.; SUMMERS, R.; ATEN, B. Penn World Table Version 6.1 Center for International Comparisons at the University of Pennsylvania (CICUP), oct. 2002.

HUBER, P., J. Robust statistics. New York: Wiley, 1981.

JONES, C. Time series tests of endogenous growth models. Quarterly Journal of Economics, n. 110, p. 495-525, 1995.

KNELLER, R.; STEVENS, P. A. The Specification of the Aggregate Production Function in the Presence of Innefficiency. Economic Letters, n. 81, p. 223-226, 2003.

KYRIACOU, G. Level and Growth Effects of Human Capital., Working Paper, 91, C.V. Starr Center, 1991. 26p.

LICHTENBERG, K. R. Have international differences in educational attainment levels narrowed?, In: BAUMOL,W. J; NELSON, R.R; WOLFF, E.N. (eds) Convergence of Productivity, Cross-National Studies and Historical Evidence. Oxford: University Press, 1994. p. 225-242.

LUCAS, R. E. On the Mechanics of Economic Development. Journal of Monetary Economics, n. 22, p. 3-42, 1988.

Why Doesn't Capital Flows from Rich to Poor Countries? American Economic Review, n. 80, p. 92-96, 1990.

MANKIW, N.; ROMER, D.; WEIL, D. A Contribution to the Empirics of Economic 
Growth. The Quarterly Journal of Economics, may, 1992.

MILLER, S.; UPADHYAY, M. P. The Effects of Openness, Trade Orientation, and Human Capital on Total Factor Productivity. Journal of Development Economics, v. 63, p. 399423, 2000.

MINCER, J. Schooling, Experience, and Earning. National Bureau of Economic Research. New York: Columbia University Press, 1974.

MURTHY, N. R. ; CHIEN, L. S. The Empirics of Economic Growth for OECD Countries: some New Findings, Economic Letters, n. 55, p. 425-429, 1997.

NELSON, R. ; PHELPS, E. Investiment in Humans, Technological Diffusion and Economic Growth, American Economic Review, Paper and Proceedings, n. 56, v. 2, p. 69-75, 1966.

NUXOLL, D. A. Differences in relative prices and international differences in growth rates. American Economic Review, n. 84, v. 5, p. 1423-1436, 1994.

O' NEIL, D. Education and Income Growth: Implications for Cross-Country Inequality. Journal of Political Economy, n. 103, p. 1289-1301, 1995.

PSACHAROPOULOS, G. Returns to Investiment in Education: a Global Update, World Development, n. 22, p. 1235-1343, 1994.

. The Profitability of Investiment in Education: Concepts and Methods, World Bank Working Paper, Washington, D.C., 1995.

ROMER, P. Increasing Returns and Long-run Growth. Journal of Political Economy, n. 94, p. 1002-1037, 1986. part 2, p. 71-102, 1990.

Endogenous Technological Change. Journal of Political Economy, n. 98,

SALA-I-MARTIN, X. I Just Ran Four Million Regressions, NBER Working Paper n. 6252, november, 1997.

. 15 Years of New Growth Economics: What Have We Learnt? Discussion Paper n. 0102-47, Department of Economics, Columbia University, april, 2002.

SCHULTZ, T. The Economic Value of Education, New York: Columbia University Press, 1963.

SOLOW, R. M. A Contribuition to the Theory of Economic Growth. Quarterly Journal of Economics, n. 70, v. 1, p. 65-94, 1956. (Feb).

. Technical Change and the Aggregate Production Function. Review of Economic and Statistics, n. 39, p. 312-320, 1957.

STAIGER, D.; STOCK, J. H. Instrumental variables regression with weak instruments. Econometrica, n. 65, v. (3), p. 557-586, 1997.

THE WORLD BANK GROUP/ ECONOMIC GROWTH RESEARCH. Disponível em: http://www.worldbank.org/research/growth/GNDdata.htm. Acesso em: 12/out/2002.

WELCH, F. Education in Production. Journal of Political Economy, n. 78, p. 35-59, 1970.

WÖBMANN, L. Specifying Human Capital: A Review, Some Extensions, and Development Effectas. Kiel Working Paper n. 1007, Kiel Institute of World Economics, Germany: Kiel, 2000.

WOOLDDRIDGE, J. M. Econometric analysis of cross section and panel data, MIT Press, 2002. 
WU, D., M. Alternative tests of independence between stochastic regressors and disturbances. Econometrica, n. 41, v. 4, p. 733-750, 1973.

WOLFF, E. Capital Formation and Productivity Growth over the Long-term. American Economic Review, n. 81, p. 565-579. 
